\title{
SPETA: Social pervasive e-Tourism advisor
}

\section{Angel García-Crespo ${ }^{\mathrm{a}, *}$, Javier Chamizo ${ }^{\mathrm{a}}$, Ismael Rivera ${ }^{\mathrm{b}}$, Myriam Mencke ${ }^{\mathrm{a}}$, Ricardo Colomo-Palacios ${ }^{a}$, Juan Miguel Gómez-Berbís ${ }^{a}$}

${ }^{a}$ Escuela Politécnica Superior, Universidad Carlos III de Madrid, Avda. de la Universidad 30, Leganés, Madrid, Spain

${ }^{\mathrm{b}}$ Digital Enterprise Research Institute, National University of Ireland, Lower Dangan, Galway, Ireland

\section{A R T I C L E I N F O}

\section{Keywords:}

Pervasive computing

Context-awareness

Semantic web

Reasoning

Social network

e-Tourism

\section{A B S T R A C T}

Tourism is one of the major sources of income for many countries. Therefore, providing efficient, real time service for tourists is a crucial competitive asset which needs to be enhanced using major technological advances. The current research has the objective of integrating technological innovation into an information system, in order to build a better user experience for the tourist. The principal strength of the approach is the fusion of con text aware pervasive systems, GIS systems, social networks and semantics. This paper pre sents the SPETA system, which uses knowledge of the user's current location, preferences, as well as a history of past locations, in order to provide the type of recommender services that tourists expect from a real tour guide.

\section{Introduction}

Currently, the tourism industry is demanding an ever increasing level of value added services in technologically com plete environments, which are integrated and highly dynamic. As a consequence of this circumstance, administrative and corporate bodies in the tourism industry now have to focus on the development of new infrastructures, providing citizens with access to cultural content and tourism services.

Additionally, the globalization of technologies, such as the Internet in the mid 1990s, and its subsequent reinvention as the Social Web or Web 2.0 (O'Reilly, 2005), have led to a scenario where the improvement of various e Tourism aspects has been amplified. For example, those dedicated to providing a better user experience through touristic routes scheduling and planning. Thus, in the context of Web 2.0, globalization and participation have opened up infinite opportunities for exploit ing the user's network contribution, by recommending particular preferences regarding tourism, and the opinion compari son possibilities in a typical Web 2.0 context. The current research is set within this background, and represents the fusion of some of the most important e Tourism trends: the semantics application, the Web 2.0 elements integration and the use of geolocation services and pervasive computing. As will be discussed later in the paper, by combining all of these technologies, new value added tourism services can be provided.

SPETA is a tool created for exploiting the information exposed by user preferences, based on similarities with a current user's social network profile, as well as the contextual information extracted from the user's location, determined by the user's mobile. Therefore, exploiting the processing provided by a pervasive terminal.

\footnotetext{
* Corresponding author.

E-mail addresses: angel.garcia@uc3m.es (A. García-Crespo), javier.chamizo@uc3m.es (J. Chamizo), ismael.rivera@deri.org (I. Rivera), myriam.mencke@ uc3m.es (M. Mencke), ricardo.colomo@uc3m.es (R. Colomo-Palacios), juanmiguel.gomez@uc3m.es (J.M. Gómez-Berbís).
} 


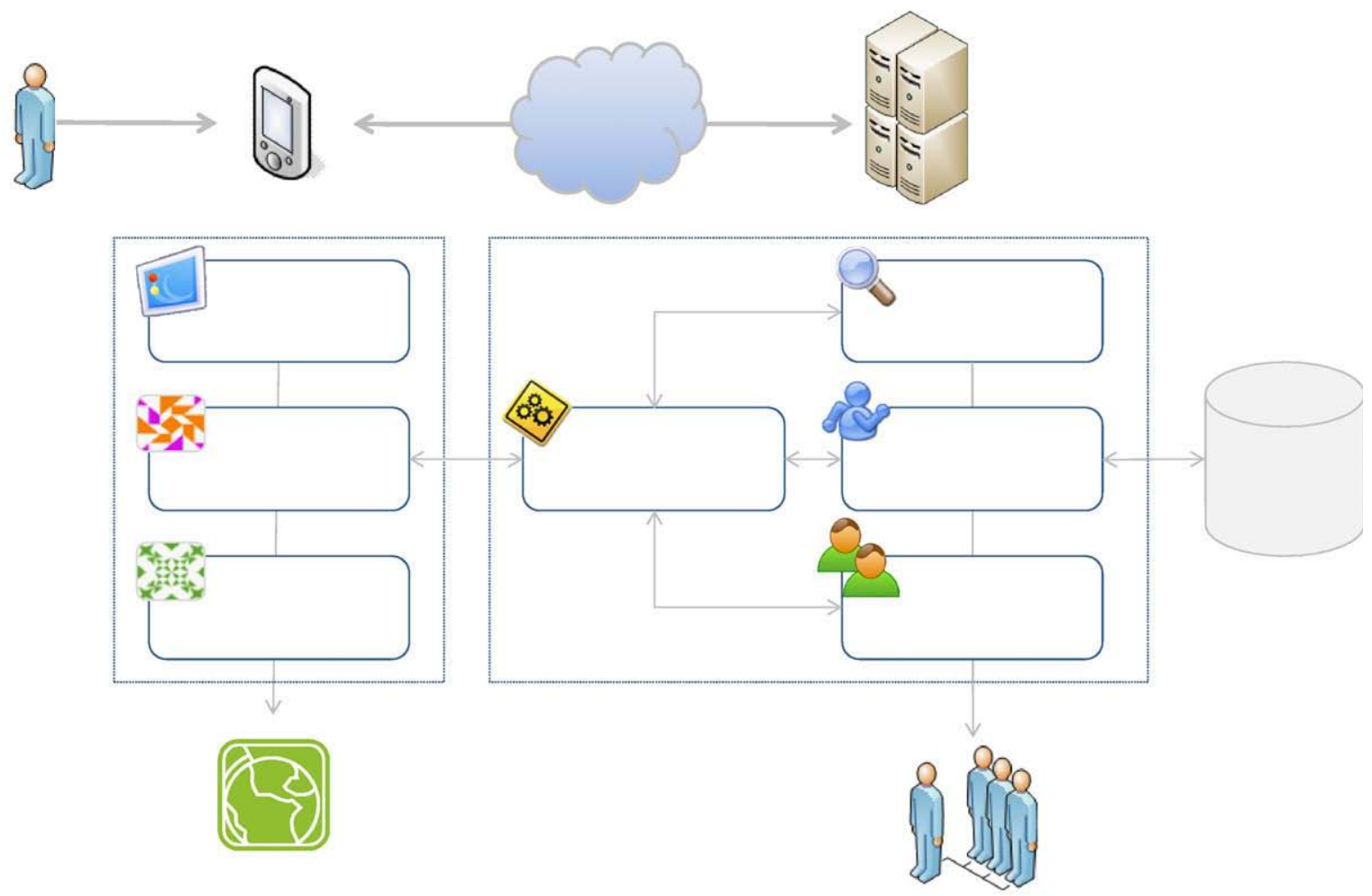

Fig. 1. SPETA architecture.

The remainder of the paper is structured as follows: Section 2 provides an overview of the state of the art in semantic based social networks and context aware pervasive systems. Section 3 presents SPETA, the solution proposed in this paper, including features, the ontology used, the algorithm proposed, the architecture and a use case. Finally, Section 4 discusses the conclusions drawn and the future work to be realized (see Fig. 1).

\section{State of the art}

The tourism context has been comprehensively studied in the application of new information technologies solutions. The great impact tourism has on society, has initiated several different approaches whose aim is to ease tourists' decision making processes.

There are several different solutions which attempt to solve the problems encountered by tourists in an unfamiliar city. Some information systems approaches try to help users by matchmaking, such as group package tours (Kanellopoulos, 2008). However, this solution does not provide any personalized guidance to users, forcing them to explore any possibilities with out guidelines.

\subsection{Recommender systems}

Recommender systems offer guidance based on users' profiles or users visiting background (Schafer et al., 2000). A rec ommender system can provide a set of solutions that best fit the user, depending on different factors concerning the user, the objective or the context where it is applied. A recent survey of recommender systems can be found in (Adomavicius and Tuzhilin, 2005).

There are different approaches to recommender systems, depending on the way algorithms derive possible recommen dations for the tourist. The most widely used approach is collaborative filtering, also known as social filtering (Breese et al., 1998). This approach focuses on the behavior of users towards items, such as purchasing habits or preferences, rather than on the nature of items the system offers.

A different approach, derived from machine learning techniques, is known as content based filtering. This approach is a concrete type of classifier system that allows the integration of widely available information into the system (Billsus and Pazzani, 1999), such as textual descriptions or image directories. It focuses on the internal item's nature, favoring the seman tics of the content over social interactions or user behavior. 
A third type of recommender system is one which uses knowledge based filtering. These systems use knowledge about users and products to reason about the possible recommendation (Burke, 2000). This approach offers a high flexibility suitable for most tasks and applications, with a strong focus on content or social semantics (Berka and Plnig, 2004). Such systems rely on an explicit representation of knowledge. In the context of e Tourism, the MAIS Project (Corallo et al., 2005) defines a knowledge based recommendation approach in an e Tourism scenario. It is based both on semantic web technologies which represent the domain, and Service Oriented Architecture for the localization of solutions. However, MAIS does not con sider users' background knowledge, since it is based on preconceived conditions for any user group, such users below the age of 25 .

There are some systems which use a combination of previous techniques to provide a recommender system (Popescuil et al., 2001). These types of algorithms are known as Hybrid Filtering. In (Berka and Plnig, 2004) a hybrid approach is defined, which applies all of the algorithms previously described in a vector like manner. The set of possible recommendations trans forms from a coarse grained set to fine grained, with the application of each type of algorithm. This system is tourism ori ented, and by the application of each different approach takes into account relevant attributes, such as temporal or economical interests.

\subsection{Context aware pervasive systems}

Schilit et al. (1994) were the pioneers of this term. Their work detailed a model of computing in which several diverse mobile and stationary systems interact with the user in order to determine, according to the user's location, objects and peo ple that are near, as well as changes in those objects over time.

Mobile systems which can sense their physical environment, in other words, their context of use, and can adjust their behavior in order to respond, are the systems which represent the context aware computing philosophy, being a part of a larger ubiquitous or pervasive computing environment. Where you are, who you are with, and what resources are nearby, are the most important aspects of the context to be considered.

Sometimes the term location aware instead of context aware is used because of the importance of location in these kinds of systems, however, location aware does not always capture interesting mobile elements, whereas context aware is used to include nearby people, devices, lighting, weather, rain probability, noise level, and even the social situation. However, this huge amount of data makes it necessary to reason on the context knowledge to solve inconsistencies of sensor data or to deduce high level situational context information from low level sensor data. Wang et al. (2004) show how ontologies can be used to solve these context modeling and reasoning issues, and demonstrate that ontologies can be used to support ontology modeling and reasoning by applying knowledge sharing, logic inference and knowledge reuse to the domain of con text in pervasive computing environments.

The possibility of offering contextual tourist information in a pervasive environment has also been broadly studied, offer ing context awareness solutions (Cheverst et al., 2002) that can guide users in a more focused direction, since only relevant information will be offered.

In this specific context Abowd et al. (1997) present a mobile context aware tour guide approach, called Cyberguide. This system was designed to assist a very specific type of tourist: a visitor undertaking a tour of the GVU Center Lab during monthly open houses. The long term goal was an application that knows where the tourist is, what he is looking at, predict ing and answering questions he might pose, providing the ability to interact with other people and the environment.

Some context aware tourist information systems offer the possibility of looking for user services. CATIS (Pashtan et al., 2003) offers an approach based on Service Oriented Architecture where the system, given the physical location of the user, offers a set of request related services in the surrounding area.

The GUIDE system (Cheverst et al., 2000) introduces a context aware tourist information approach for Lancaster, where wireless gadgets show contextual information to tourists, detailing places being visited. The solution obtains context infor mation, such as actual position, through a position transmitting stations network. This system is only able to show informa tion regarding surroundings. The system is also aware of the state of any service that could be interesting, not showing those that are closed, for example. The maintenance of a user's visiting history is another of its functionalities. It stores places vis ited or GUIDE past uses in order to be more prepared for potential future uses. It is in fact, a simple recommender and con text aware system, which is the basis of the present paper.

\section{SPETA}

SPETA is a novel and promising solution, which combines social networks, semantic Web, and context awareness in per vasive systems, created to improve the tourist experience. It offers a personalized guide and solves the tourism services dis integration, with regard to searching, finding and showing personalized services by means of semantic, geolocation, and social technologies.

Regarding the semantic Web, the solution presented is based on an ontology which stores all the knowledge required, also integrating social and geolocation information, and provides the reasoning capacity required. The advisor characteristic of the solution needs a sophisticated algorithm which, taking advantage of all of the technologies mentioned, will give the user the best recommendations. 


\subsection{Features}

The system is oriented towards people who need guidance when visiting a city they are not familiar with, and conse quently they do not know which places are interesting to visit. The appropriate discovery of tourism services is one of the main drawbacks when people travel (Hui et al., 2007). Based on the user profile, which determines the common char acteristics of the previously visited places and the user behavior, the system recommends attractions which are likely to fit the user expectations.

Fundamentally, SPETA is sensitive to six different characteristics that enable it to assist the user with accurate advice of new destinations to encounter: real time location, weather forecast, time, user preferences, friend's recommendations and history.

The first issue which must be dealt with is the user's location. To unambiguously locate the user by physical coordinates, a GIS system is used. Location and characteristics of context aware systems (Schilit, 1995) are used by the system to register the physical position of the user and any subsequent moves made. Knowing the user location, a set of places to visit can be offered, but also allows the user to detect contacts or friends in the surrounding areas.

Besides location, time and weather are also taken into account. These features will be used, for instance, to recommend indoor locations when the weather does not give any other possibility, and takes into consideration timetable restrictions of attractions.

The user profile or preferences are constructed in two ways. The first one requires explicit interaction of the user, deter mining what their interests are, what kind of places they prefer to visit, and the ratings given to attractions. Additionally, a huge amount of information can be extracted from the social networks they belong to, such as favourite painters, writers, or music preferences. Usage of the system provides feedback to the system itself, in other words, user behavior is taken into consideration. For example, the type of museums that are always visited by the user or his preferences regarding parks and gardens to spend the evening, etc.

The system uses the power of third party social networks to extract information about the user. The central information stored in a social network is the user's contact data. All the user's contacts are extracted and integrated into the system pro posed. Usually, people consult their trusted friend network prior to travelling to obtain travelling recommendations. This feature is enabled by taking into consideration the contact's attraction ratings.

The social networks used in this work are applied adopting the OpenSocial ${ }^{1}$ API (Application Programming Interface). Thus, a new tourism oriented semantic based social network is created as part of the system. Within the Open Social APIs, in the cur rent work are only the following elements are used: People, which give information about individual people and their relation ships to each other; and Activities, which provide updates regarding what people are doing.

\section{2. e Tourism ontology}

In knowledge management and semantic web research areas, ontologies are considered essential in order to describe the concepts and relationships between them. They provide a way to achieve integration and interoperability through the use of a shared vocabulary and meanings for terms.

Ontology contains the classes of entities, relations between them and the axioms, which apply to the entities of that domain:

- Classes and instances. The classes are the abstract representation of the entities such as 'City', 'Artist', 'Restaurant', or 'Accommodation'. However, an instance is a concrete representation of a class: 'Madrid', 'Pablo Picasso', 'Asador Don ostiarra', or 'Hotel Ritz'.

- Properties. A property establishes relationships between the classes of ontology. For instance, the property 'locatedIn' asso ciates a location to a place.

- Rules. They are the axioms of ontology, and model logical sentences which are always true. Rules make high expressive ness and complex reasoning available.

As part of this work, an ontology which represents the tourism domain is required. Tourism is a data rich domain, and depending on the purpose of the study, different approaches can be considered. For an intelligent advisor as that proposed by the current system, recommendation aspects also have to be strongly considered. As a conclusion, the defined ontology integrates tourism specific domain concepts and recommendation characteristics.

The ontology has been built to answer three main questions (Cardoso and Sheth, 2006): what a tourist can view and visit; where the interesting places to see and visit are located; and when can a tourist visit a particular place. However, the ontol ogy proposed in this work will also answer the following questions: which friends are visiting (or will visit) a place; and what a tourist can do in a specific place. Hence, the main concepts modelled in the ontology are the super classes 'Activity', 'Attraction', 'Place', and 'Person'.

\footnotetext{
${ }^{1}$ http://code.google.com/apis/opensocial/.
} 
It is important to emphasize that a Person is not just a tourist. The concept Person also covers all kinds of people such as film directors, famous painters, etc. A person is a common concept in many cases, so there are a myriad of approaches which deal with people and the relationships between them. The Friend of a Friend (FOAF) project ${ }^{2}$ has created a vocabulary to de scribe people, the links between them and the things they create and do, in other words, by adopting FOAF, the problem con cerning the defining of the tourist, who his friends are, and all the social links around him, is resolved.

Furthermore, in order to represent the places, attractions and activities a tourist can visit or enjoy a number of projects are taken into account. The DBpedia ${ }^{3}$ integrates the information contained in Wikipedia, the largest public encyclopaedia on the Web, with semantic Web technologies, enabling powerful queries against a structured and interlinked set of information. Consequently, the DBpedia dataset describes more than 2.49 million "things", including at least 108,000 persons, 392,000 places and 36,000 films, in a structured manner. It contains 588,000 links to images, 3,150,000 links to relevant external web pages, 2,180,000 external links to other datasets, 207,000 Wikipedia categories, and 75,000 YAGO ${ }^{4}$ categories. YAGO is a huge semantic knowledge base, and it has a lot of very useful categories and properties for the tourism domain, such as 'locatedIn', 'interestedIn' 'hasCurrency', etc. Moreover, the DBpedia dataset contains geo coordinates for 392,000 geographic locations. Geo coordinates are expressed using the W3C Basic Geo Vocabulary. ${ }^{5}$ It is possible to get the geo coordinates of a specific attraction, but it also allows permits the obtaining of all of the elements near that attraction, specifying a distance. Summarising, the concepts Activity, Attraction, Place and Person are covered, and the ontology is also populated with a huge amount of instances. With the vocabularies and ontologies adopted, it is possible to answer all of the questions previously raised.

Despite dealing with most of the concepts involved, including attractions, the authors of this paper considered it impor tant to increase the knowledge regarding attractions in order to enhance the recommendation algorithm, deciding that the inclusion of a taxonomy distinguishing the attractions was necessary. A list of the main categories of the taxonomy is shown below:

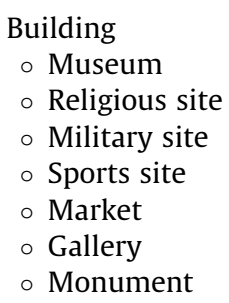

- Historical monument

- Modern monument

Entertainment

$\circ$ Restaurant

- Amusement park

- Water park

- Leisure centre

- Cinema

- Theatre

- Casino

- Craft centres

- Festivals and pageants

Archaeological place

Landscape

- Treasured landscape

- National forest

- Zoos \& Aquariums

- Park

- Natural park

- National park

- State park

- Countryside

- Garden

- Waterfall

\footnotetext{
${ }^{2}$ http://www.foaf-project.org/.

${ }^{3}$ http://wiki.dbpedia.org/.

${ }^{4}$ http://www.mpi-inf.mpg.de/ suchanek/downloads/yago/home.htm.

${ }^{5}$ http://www.w3.org/2003/01/geo/.
} 
This taxonomy has been completed taking into account the different categories of museums, the list of archaeological periods associated with archaeological sites, and so on. Moreover, elements such as attractions associated with historic peo ple are not required to be represented in the taxonomy, because they can be modelled as an attraction related to a historical figure, using the properties from DBpedia and YAGO.

Regarding the attractions, three more aspects have to be considered. When a tourist can visit a place, what is the attrac tion theme (culture, leisure, modern art, etc.), and where the attraction is located (indoor or outdoor), in relation to weather constraints. Timetable constraints can be defined by the following assumptions and rules:

- An attraction is always open: $24 \times 7$.

- The rule closeBetween(?attraction, ?timeClose, ?timeOpen) specifies the closed time of the attraction every day.

- The rule closeOnDate(?attraction, ?date) specifies that the attraction is closed for the whole day. This rule can be used for example to specify if a specific museum closes every Tuesday: closeOnDate(Prado, ?date) $\mathcal{E}$ ?date == Tuesday.

The attraction theme is specified through a new property called 'theme', and each attraction has the property 'isIndoor', to specify whether the attraction is indoor or outdoor.

\subsection{Recommender algorithm}

The way the recommender system calculates which services are suitable for a particular user depends on many factors in a tourism context. The location, weather, interests, historical visits and even the time the query is made are relevant. With such a complex decision making structure, a robust algorithm is needed, which must be able to process these heterogeneous variables.

The SPETA system is based on a hybrid filtering approach (Baudisch, 1999; Berka and Plnig, 2004) divided into four mod ules ordered in a row like structure that filters the possible services to recommend and order them, according to which one is most likely to interest the user. This hybrid approach makes use of a variety of techniques which include context aware, knowledge based and social based methods to retrieve the most suitable services.

The first step the system takes is to filter the possible results based on contextual information of the pervasive system, such as the location, the time or the weather. While location is a prime determinant of what information should be retrieved, time or weather can also affect the suggested information content that is delivered. This process provides an initial selection of services, based on simple database interactions. This yields a very efficient reduction of the number of items, which have to be processed, at a very early stage of the workflow.

In order to determine which services are located in an affordable position, a sliding grid window methodology is devised. This methodology takes into account location, speed and direction of travel. Any element outside the resulting window will be filtered, so that the system only considers those that are at reachable position. Therefore, the speed determines the win dow range. Also, the direction of travel can be considered, narrowing the window of the elements on the opposite direction of the movement.

Once one preliminary set of services is obtained, the process can continue in order to obtain a more fine grained set of results. The second module applies Knowledge based filtering techniques to obtain a more concise set of services, taking into account the semantics associated with them. Both the user and the services are expressed in ontology like structures which allow the application of feature based similarity algorithms (Paolucci et al., 2002). This approach is extended in order to evaluate the semantic similarity on n different ontologies that result useful when comparing users' preferences with services descriptions. The result of the application of such algorithm will be a number between 0 and 1 that defines the semantic similarity between user preferences and touristic service. A threshold will be applied, filtering any element under the defined threshold and storing the resulting value of each service.

At this point, each service will have a vector defined, known as result vector, where the result of each of the algorithms to be applied will be stored. This is needed to evaluate which of the remaining services is more suitable for a user.

Although user interests and context information could already give a relatively good set of results, it is important to take into account social information with regard to other people's experiences and also user's past experiences. Consequently, the third module applies collaborative based filtering techniques to the remainder of the services.

The user preferences can be represented as a vector, where each element represents one possible preference in the system; for example, Chinese food. These preferences can evolve depending on the places visited in an non complex way; if a user usually goes to Chinese restaurants, the element on the vector representing the Chinese food will become larger and larger; on the contrary, if a user each time goes less frequently to a Chinese restaurant and starts liking Mex ican food, the element representing Chinese food will decrease, while the Mexican food preference element will start increasing. These vectors are independent from the semantic description of preferences used in the second module; in fact, vectors are an efficient solution to continuous learning.

If each service is also represented as a vector, vector based classifying techniques can be used to calculate the distance between vectors and identify which services are more suitable for a given user. Support vector machines technique (Man ning and Schütze, 1999) allows the classification of information expressed by vectors in an efficient way, also calculating the distance between the different elements. The larger the distance between the vector representing user preferences and a 
vector representing a service, the less suitable it is for the user. The result vector will store the inverse of the distance, there fore introducing which services are the nearest ones to a user interest.

In a social network, any user has relationships with friends who usually share his interests. Based on that concept, the algorithm takes into account if a service has been visited by a friend previously, and what assessment was made. Since two friends do not need to have similar interests, a semantic similarity evaluation must be made between the user and his friend's preferences in order to obtain a weight to apply to the assessment value mentioned. The semantic evaluation is previously stored since it is nearly static and does not change with time, so the process only takes the multiplication of the similarity weight and the assessment value given by the friend.

After all of the processing, each service has some values attached in the result vector that describe how well a service compels the user interests. Thus, the fourth module orders the different services according to the information obtained in previous module. Each value in the vector will have an attached weight according to the importance it has in the overall pro cedure, determined by subjective means. For instance, it is more important that a service is semantically similar to some user preferences; than it is that any friend has visited it. As a result, the algorithm will offer only those services that can fulfil user needs given both contextual and personal factors.

\subsection{Architecture}

Since any architecture heavily relies on the software component structure and its relationships, a number of architectural decisions which included designing a distributed system were made, composed of a number of pervasive modules. On the one hand, lightweight thin client style pervasive modules are located on the user side (i.e. software components with less requirements and constraints in terms of performance, quality of service or computational capacity). On the other hand, the knowledge and reasoning side is stored and managed from the server software infrastructure. The architecture of the SPETA system is composed of the modules depicted in the figure which follows.

These components will be introduced as follows:

- GUI: this is the component that interacts with the user. It is located in the pervasive module, since the system will only interact with the user through this module. It shows information collected by the different means previously explained, such as physical location, historical knowledge, preferences or social links. It also offers the possibility to look directly for any service or any kind of service the user is interested in, in a given moment.

- GIS: the GIS module is in charge of unambiguously locating the user's physical position and translating it into a logical position over a map, used to look for surrounding services. The system initially uses GPS satellite systems to locate the pervasive device and to provide the physical coordinates. Subsequently, those coordinates will be used to establish the logical location (city, street and place) where the user is using the system. Maps are downloaded from the internet; there fore they give the user a visual output of his surroundings, indicating possible tourism services the user could be inter ested in.

- Managers: manager components are the main components of the architecture. They control the different interactions among the components. The pervasive Manager communicates both with the GIS system (to establish its position and retrieve a map of the location) and with the GUI system, in order to show any relevant information and interact with the user. The server Manager communicates with the social network to get any relevant user preferences of his environ ment; it communicates with the service locator to get all relevant tourism services given a set of constrains that filters all the set of services; and finally it also communicates with the reasoner in order to manage historical knowledge from the user.

- Service locator: this module offers all of the functionality to locate and obtain any pertinent information about tourism services. The system offers the possibility of retrieving only those services that fit with the environment, illustrated as constraints, where the user is interacting. The service locator interacts with the reasoner where all services installed knowledge is handled in the system. As outlined before, all knowledge is stored in ontologies that will be managed through the reasoner module. The ontology for tourism services will be based on the e Tourism (Cardoso and Sheth, 2006).

- Social network: the social network manages all knowledge derived from the user and his network connections with friends or members from the same interest groups. The module is in charge of obtaining all the constraints involved in the useŕs social relations. The pervasive module will also interact with the social network, keeping track of the place the members are, and providing support for personal interaction.

- Reasoner: this module is the responsible for managing, storing and inferring over all the system knowledge. Although knowledge managed by the system has heterogeneous origins, all of it is closely related. Hence, the storage of the knowl edge had to be in the same source so that it could be interrelated and inference processes could deal with the whole knowledge base, avoiding the drawbacks introduced by distributed data. The reasoner will be discussed in more detail in further sections, since it is considered that the semantic section of the project deserves a more comprehensive analysis of its design and usefulness.

Essentially, the reasoner module supports the other components efficiently by retrieving, managing and storing any infor mation in the knowledge base. Consequently, the system gains reasoning capabilities by means of an entailing procedure, which derives knowledge from that explicitly indicated in contextual ontologies and defining preferences and constraints. 
Additionally, it provides capabilities to add, modify, append or eliminate explicit knowledge stored in the system. Specific facts from this knowledge base might provide feedback to any user needs based on the resulting semantic networks that link preferences, history, friends and any other useful information.

After a preliminary analysis of the reasoner, the authors determined that the reasoning module needed to be installed in a remote system, because of the computational requirements of the reasoning process (Tempich and Volz, 2003; Tsarkov and Horrocks, 2003), and because it is intended to be a centralized system to share knowledge between users. Since the system is expected to be installed in heterogeneous pervasive components, the computational capacities can also vary depending on the component that uses the system. Due to the necessity of having a remote module for the reasoning process, the storage of all ontologies managed by the system was an intrinsic requirement, since it improved performance by reducing network traffic. Therefore, the reasoner also had to provide a set of tools to allow accurate ontology management and the knowledge stored; permitting operations such as querying, insertion or deletion.

\subsection{Use case}

This section will show a typical use case scenario where we can place SPETA functionalities, showing an advancement in interactive and touristic systems.

In Fig. 2 is shown a scenario where two different users of the system have the same initial state. Both John Smith and Robert Davies will step out of the Prado Museum and at that moment they will consult their pervasive devices, to get a new itinerary. It must be pointed out that both users have heterogeneous interests, meaning different preferences.

Once they have stepped out of the museum, SPETA will detect their positions by means of the GIS module, which will communicate with the service locator. Since the previous state was inside the museum, the system infers that both users have already left, and starts reasoning to determine some new potential tourism services.

SPETA accesses preference information about each user, obtaining that John is a botanical lover and a vegetarian; on the other hand, the system detects that Robert is a gourmet and prefers contemporary, trendy locations. Given that information gathered from the social network, the system also checks temporal constrains, weather forecast and historical knowledge about previous moves.
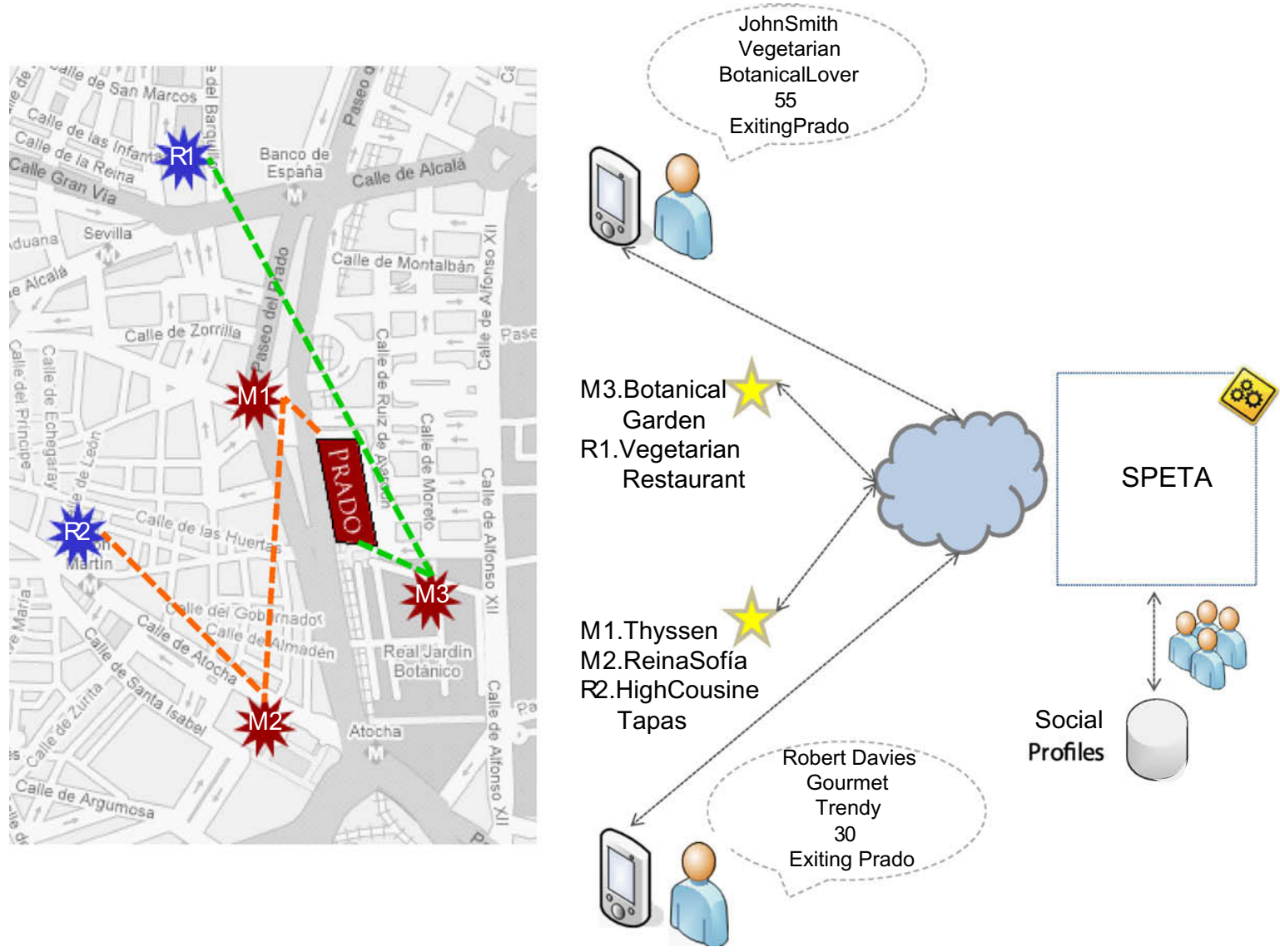

Fig. 2. Use case. 
As a result, SPETA gets a complete set of constrains that will be used to query on the service locator to get a complete itinerary that fits best with each user preferences.

The system takes into account that John loves vegetables and the fact that the botanical garden is next to his location, recommending him to visit it. Furthermore, temporal and historical variables are considered. SPETA is aware that John usu ally has lunch at 13:30 and since the time of the query was 13:00, it also offers a vegetarian restaurant in the area, in case he is hungry.

In the case of Robert, the system takes into account that he is a trend seeker and offers him some modern art museums in the surroundings. As in John's case, the time of the day is considered to offer an upmarket restaurant to have lunch.

\section{Conclusions and future work}

Tourism has gained momentum as a top priority domain challenge for cutting edge technologies. The impact of its appli cation has been rapidly seized by public and private institutions, in order to effectively gain a competitive advantage. e Tour ism has then emerged as a promising revision of the previous business models and initiatives related to tourism, with the advent of a new breed of technologies encompassed by the Web 3.0 buzzword, such as Geographical Information Systems, Recommendation and Semantic Technologies and Pervasive Computing.

In this work, a novel approach to achieve the creation of a framework has been presented in which contextual recommen dations can be realized, taking advantage of the characteristics of Web 3.0 technologies. The SPETA framework provides a fully fledged platform and architecture and a proof of concept implementation which presents, on one hand, the combining of the mentioned cutting edge technologies and emerging standards; and on the other, reflects research efforts and innovation.

Our future work with regards to SPETA encompasses three different research directions: Firstly, the mathematical anal ysis of the behavior of platform users, as a powerful mechanism to recommend, support and advice, to improve the user experience. Secondly, considering potential offline developments that could resume the system activity without suffering from network disconnection. Finally, multi device personalization and profiling is envisaged as the next step towards a reli able pervasive application which benefits from current technology possibilities.

\section{Acknowledgements}

This work is supported by the Spanish Ministry of Industry, Tourism, and Commerce under the GODO project (FIT 3400002007 134), under the PIBES project of the Spanish Committee of Education and Science (TEC2006 12365 C02 01) and under the MID CBR project of the Spanish Committee of Education and Science (TIN2006 15140 C03 02).

\section{References}

Abowd, G.D., Atkeson, C.G., Hong, J., Long, S., Kooper, R., Pinkerton, M., 1997. Cyberguide: a mobile context-aware tour guide. ACM Wireless Networks 3 (5), 421-433.

Adomavicius, G., Tuzhilin, A., 2005. Toward the next generation of recommender systems: a survey of the state-of-the-art and possible extensions. IEEE Transactions on Knowledge and Data Engineering 17 (6), 734-749.

Baudisch, P., 1999. Joining collaborative and content-based filtering. In: Interacting with Recommender Systems, Online Proceedings of the CHI '99 Workshop.

Berka, T., Plnig M., 2004. Designing recommender systems for tourism. In: The 11th International Conference on Information Technology in Travel and Tourism, ENTER, Cairo, Egypt.

Billsus, D., Pazzani, M., 1999. A hybrid user model for news story classification. In: Proceedings of the Seventh International Conference on User Modeling, Banff, Canada, pp. 20-24.

Breese, J.S., Heckerman, D., Kadie, C., 1998. Empirical analysis of predictive algorithms for collaborative filtering. In: Proceedings of the 14th Conference on Uncertainty in Artificial Intelligence, pp. 43-52.

Burke, R., 2000. Knowledge-based recommender systems. In: Kent, A. (Ed.), Encyclopedia of Library and Information Systems, vol. 69. Marcel Dekker, New York, NY.

Cardoso, J., Sheth, A., 2006. Developing an owl ontology for e-Tourism. In: Cardoso, J., Sheth, A. (Eds.), Semantic Web Services, Processes and Application. Springer, New York.

Cheverst, K., Davies, N., Mitchell, K., Friday, A., Efstratiou, F., 2000. Developing a context-aware electronic tourist guide: some issues and experiences. In: Proceedings of the SIGCHI Conference on Human Factors in Computing Systems, April 01-06, The Hague, The Netherlands, pp. 17-24.

Cheverst, K., Mitchell, K., Davies, N., 2002. The role of adaptive hypermedia in a context-aware tourist GUIDE. Communications of the ACM 45 (5), $47-51$.

Corallo, A., Lorenzo, G., Solazzo, G., 2005. A semantic recommender engine enabling an e-Tourism scenario. In: Proceedings of the International Semantic Web Conference.

Hui, T.K., Wanand, D., Alvin, H., 2007. Tourists' satisfaction, recommendation and revisiting Singapore. Tourism Management 28 (4), $965-975$.

Kanellopoulos, D.N., 2008. An ontology-based system for intelligent matching of travellers' needs for group package tours. International Journal of Digital Culture and Electronic Tourism 1 (1), 76-99.

Manning, C., Schütze, H., 1999. Foundations of Statistical Natural Language Processing. MIT Press, Cambridge, MA.

O'Reilly, T., 2005 What is web 2.0?. O'Reilly NetWork. Retrieved October 16, 2008, from <http://www.oreillynet.com/pub/a/oreilly/tim/news/2005/09/30/ what-is-web-20.html>.

Paolucci, M., Kawamura, T., Payne, T.R., Sycara, K.P., 2002. Semantic matching of web services capabilities. In: ISWC'02: Proceedings of First International Semantic Web Conference on the Semantic Web, London, UK, pp. 333-347.

Pashtan, A., Blattler, R., Heusser, A., Scheuermann, P., 2003. CATIS: a context-aware tourist information system. In: Proceedings of IMC 2003, 4th International Workshop of Mobile Computing, Rostock, Germany.

Popescuil, A., Ungar, L., Pennock, D., Lawrence, S., 2001. Probabilistic models for unified collaborative and content-based recommendation in sparse-data environments. In: Proceedings of the 17th Conference on Uncertainty in Artificial Intelligence, Seattle, WA.

Schafer, J.B., Konstan, J., Riedl, J., 2000. Recommender systems in E-commerce. In: Proceedings of the first ACM Conference on Electronic Commerce, pp. 158-166. 
Schilit, W.N., 1995. System architecture for context-aware mobile computing, Ph.D. Thesis, Columbia University.

Schilit, B., Adams, N., Want, R., 1994. Context-aware computing applications. In: IEEE Workshop on Mobile Computing Systems and Applications (WMCSA'94), Santa Cruz, CA, pp. 89-101.

Tempich, C., Volz, R., 2003. Towards a benchmark for semantic Web reasoners-an analysis of the DAML ontology library. In: Workshop on Evaluation on Ontology-based Tools, ISWC.

Tsarkov, D., Horrocks, I., 2003. DL Reasoner vs. first-order prover. In: Proceedings of the 2003 Description Logic Workshop.

Wang, X.H., Zhang, D.Q., Gu, T., Pung, H.K., 2004. Ontology based context modeling and reasoning using OWL. In: Pervasive Computing and Communications Workshops in Proceedings of the Second IEEE Annual Conference, pp. 18-22. 